CLINICAL RESEARCH ARTICLE

\title{
Cerebellar injury in term neonates with hypoxic-ischemic encephalopathy is underestimated
}

Kim V. Annink ${ }^{1}$, Lilly Meerts ${ }^{1,2}$, Niek E. van der Aa ${ }^{1}$, Thomas Alderliesten ${ }^{1}$, Peter G. J. Nikkels ${ }^{3}$, Cora H. A. Nijboer ${ }^{2}$, Floris Groenendaal ${ }^{1}$, Linda S. de Vries ${ }^{1}$, Manon J. N. L. Benders ${ }^{1}$, Freek E. Hoebeek ${ }^{2}$ and Jeroen Dudink ${ }^{1}$

BACKGROUND: Postmortem examinations frequently show cerebellar injury in infants with severe hypoxic-ischemic encephalopathy (HIE), while it is less well visible on MRI. The primary aim was to investigate the correlation between cerebellar apparent diffusion coefficient (ADC) values and histopathology in infants with HIE. The secondary aim was to compare ADC values in the cerebellum of infants with HIE and infants without brain injury.

METHODS: ADC values in the cerebellar vermis, hemispheres and dentate nucleus (DN) of (near-)term infants with HIE ( $n=33$ ) within the first week after birth were compared with neonates with congenital non-cardiac anomalies, normal postoperative MRIs and normal outcome $(n=22)$. Microglia/macrophage activation was assessed using CD68 and/or HLA-DR staining and Purkinje cell (PC) injury using H\&E-stained slices. The correlation between ADC values and the histopathological measures was analyzed. RESULTS: ADC values in the vermis $(p=0.021)$ and DN $(p<0.001)$ were significantly lower in infants with HIE compared to controls. $A D C$ values in the cerebellar hemispheres were comparable. ADC values in the vermis were correlated with the number and percentage of normal PCs; otherwise ADC values and histology were not correlated.

CONCLUSION: Histopathological injury in the cerebellum is common in infants with HIE. ADC values underestimate histopathological injury.

Pediatric Research _\#\#\#\#\#\#\#\#\#\#\#\#\#\#\#\#\#\#\#; https://doi.org/10.1038/s41390-020-01173-z

\section{IMPACT:}

- ADC values might underestimate cerebellar injury in neonates with HIE.

- $\mathrm{ADC}$ values in the vermis and dentate nucleus of infants with HIE are lower compared to controls, but not in the cerebellar hemispheres.

- Abnormal ADC values are only found when cytotoxic edema is very severe.

- $A D C$ values in the vermis are correlated with Purkinje cell injury in the vermis; furthermore, there were no correlations between $A D C$ values and histopathological measures.

\section{INTRODUCTION}

Despite therapeutic hypothermia, hypoxic-ischemic encephalopathy (HIE) following perinatal asphyxia is still an important cause of mortality and morbidity in term-born neonates. ${ }^{1}$ HIE can lead to different long-term neurodevelopmental deficits, such as motor impairment, cognitive and behavioral problems, and epilepsy. ${ }^{1-3}$ Future neurodevelopmental outcome can be predicted based on early injury patterns seen on neuroimaging. ${ }^{4,5}$ Magnetic resonance imaging (MRI) is a very accurate and reliable neuroimaging technique to visualize supratentorial patterns of hypoxic-ischemic brain injury. ${ }^{5,6}$ Especially, diffusion weighted imaging (DWI) is a frequently used MRI sequence in $\mathrm{HIE}$, which has shown to be accurate in detecting brain injury in infants with HIE. ${ }^{7}$ DWI can visualize the diffusion restriction of water molecules caused by acute cytotoxic edema. ${ }^{8}$ Furthermore, diffusion restriction can be quantified by lower apparent diffusion coefficient (ADC) measurements. ${ }^{6}$ Research until now has shown that supratentorial DWI is very well correlated with histopathology. ${ }^{9}$ Furthermore, $A D C$ values of the basal ganglia and thalami are predictive for neurodevelopmental outcome in HIE. ${ }^{10,11}$

During the past decades, the main focus of neuroimaging in $\mathrm{HIE}$ has been on the supratentorial brain structures. However, recent literature has emphasized the vulnerability of the developing cerebellum for hypoxic-ischemic events. ${ }^{12,13}$ The cellular and network organization in the cerebellum change rapidly in the third trimester and first months after birth. ${ }^{14-16}$ The cerebellar cortex receives afferent inputs from the cerebrum, spinal cord, and vestibular nuclei via mossy and climbing fibers. ${ }^{17,18}$ The Purkinje cells (PC) are the only output neurons of the cerebellar cortex ${ }^{19}$ and are connected to the cerebellar nuclei (the largest is the dentate nucleus (DN)), which predominantly via thalamus are connected back to the cerebral cortex. ${ }^{18}$

\footnotetext{
'Department of Neonatology, Wilhelmina Children's Hospital, University Medical Center Utrecht Brain Center, University Utrecht, Utrecht, The Netherlands; ${ }^{2}$ Department of Developmental Origins of Disease, University Medical Center Utrecht Brain Centre, University Utrecht, Utrecht, The Netherlands and ${ }^{3}$ Department of Pathology, University Medical Center Utrecht, University Utrecht, Utrecht, The Netherlands

Correspondence: Jeroen Dudink (j.dudink@umcutrecht.nl)
}

Received: 20 May 2020 Revised: 21 August 2020 Accepted: 2 September 2020

Published online: 23 September 2020 
Historically, the cerebellum is especially known for its role in motor control because cerebellar damage leads to impairments in motor control and posture. However, it has become apparent that the cerebellum also plays an important role in various cognitive functions. $^{20,21}$ Multiple studies have shown that there is an association between peripartum cerebellar injury and autism spectrum disorders in preterm born infants. ${ }^{22}$ In a meta-analysis including preterm born infants, hemorrhages in the vermis led to cognitive problems in $80 \%$ of the infants. ${ }^{23}$ Nevertheless, studies about cerebellar injury on MRI in term neonates with HIE are scarce. Clinical MR sequences as DWI and T1-weighted imaging found little cerebellar injury in neonates with HIE. ${ }^{10,24,25}$ More advanced MRI studies have identified cerebellar injury in HIE using diffusion tensor imaging (DTI) in the first month after birth. Also during follow-up, between 5 and 19 months, atrophy of the vermis was seen in children with perinatal HIE on conventional imaging. $^{21,24}$

Only a few postmortem histopathological studies have been performed, which all showed extensive cerebellar injury in infants with severe HIE. ${ }^{9,12,25}$ Especially PCs appear to be vulnerable to hypoxia, which leads to altered morphology or necrosis of these cells following perinatal hypoxia. ${ }^{13,26,27}$ Also the cerebellar nuclei, e.g. DN, are vulnerable and this is probably important for cerebellar functioning, since the connections between the cerebellar hemispheres and cerebral cortex converge through these nuclei. ${ }^{28}$

There seems to be a discrepancy in cerebellar injury in infants with HIE based on MRI and histology. It is important to elucidate whether DWI can reliably diagnose cerebellar injury in infants with $\mathrm{HIE}$, because cerebellar injury might be associated with behavioral and cognitive problems. Therefore, the primary aim of this study was to investigate the correlation between the cerebellar ADC values in the vermis, cerebellar hemispheres and DN on DWI and the extent of histological cerebellar injury (cell death and inflammation) in infants with HIE. The secondary aim was to compare ADC values in the cerebellum of infants with HIE to neonates without brain injury.

\section{METHODS}

Study population

In this retrospective study, we included (near-)term-born neonates diagnosed with $\mathrm{HIE}$, who died in the neonatal period and of whom histological material was available and underwent an antemortem DWI of the brain. All infants had to fulfill the therapeutic hypothermia inclusion criteria, although half of them were born before therapeutic hypothermia became standard of care in 2008. ${ }^{1}$ Exclusion criteria were a gestational age $<36$ weeks, suspected genetic abnormalities, absence/poor quality of histological material of the cerebellum, or absence/poor quality of the DWI scan.

For the analysis of the DWI scans, we also included a group of neonates with congenital non-cardiac anomalies, without underlying syndromes, with a normal postoperative MRI (range 3-13; median 7.5 days after birth) and normal neurodevelopmental follow-up at 18-24 months of age as a "control group".

Parental consent for postmortem histopathological examination of the brain was obtained for all patients. This study was approved by the medical ethical committee (\#18-167) as a "non-Medical Research Act" study and the Biobank of the University Medical Centre Utrecht approved to use the rest biomaterial for this research (biobank \#18-284).

Magnetic resonance imaging

MRI was conducted in the first week after birth in the HIE group and within the first 2 weeks after birth in the control group using a 1.5 or 3.0 Tesla (T) MRI scanner (Philips Medical Systems, Best, The Netherlands). Standard scan protocols included at least axial DWI

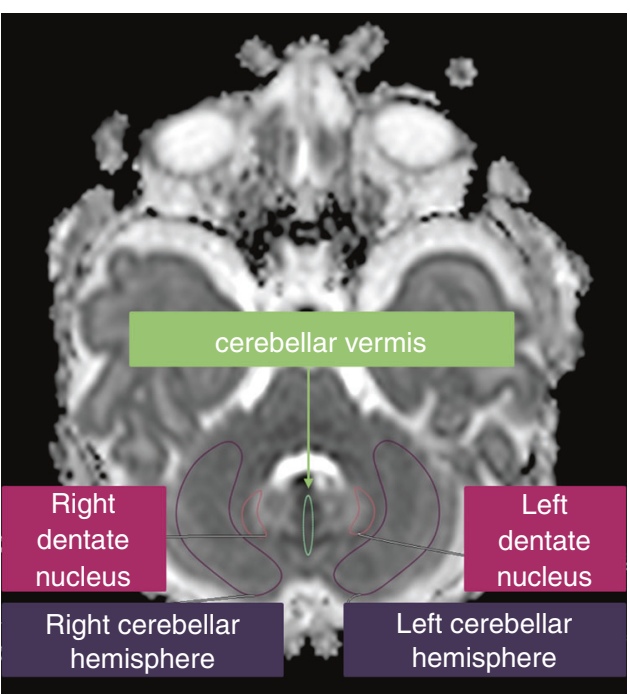

Fig. 1 Region of interest template. The template for ADC measurements at the level of the pons and vermis that was copied to all scans and slightly adapted per patient.

and T1-weighted and T2-weighted images. ADC maps were created as described in Alderliesten et al. ${ }^{10}$ The slice thickness was $4 \mathrm{~mm}$ for both 1.5 and 3.0 T MRI.

ADC values were measured in the vermis, cerebellar hemispheres, and the left and right DN using Horos Imaging software (The Horos Project, available at https://www.horosproject.org). A region of interest $(\mathrm{ROI})$ template was developed and copied to all scans (Fig. 1). The ROI was manually adapted per patient if necessary, because of the anatomical variances between patients. The anatomy was verified using T1-weighted and T2-weighted MR images and inclusion of cerebrospinal fluid in the ROI was avoided at all times. ADC values were automatically measured within the ROI. Two independent raters (K.V.A. and L.M.) measured ADC values in all patients to analyze interrater variability.

\section{Postmortem analyses}

The histopathological examination of the brains of the infants with HIE was performed in the UMC Utrecht using the following protocol: sections of various brain regions, including cerebellum and vermis, were obtained after removing and fixing the brains in $4 \%$ buffered formalin for 3-4 weeks. After fixation, a transverse section of the vermis and central section of the cerebellar hemisphere with DN were sampled and embedded in paraffin. Histological sections of 5-6 $\mu \mathrm{m}$ thick were cut and mounted on coated slides. Slides were stained with hematoxylin and eosin (H\&E) or with monoclonal antibodies against CD68 (Novo Castra, Newcastle, UK) and HLA-DR (Dako, Glostrup, Denmark). The H\&E staining was used to study the morphology and number of PCs. The CD68 and HLA-DR stains were used to visualize microglia and macrophages activation. ${ }^{13}$ The tissue was photographed and analyzed using a standard light microscope (Zeiss AXIO Lab.A1, Oberkochen, Germany) and the ZEN 2.3 Lite software (Zeiss).

H\&E staining-scoring Purkinje cells

Pictures were taken at six different locations in the vermis and cerebellar hemispheres: (1) at the apex and (2) the base of the anterior lobe, (3) at the apex and (4) the base of the posterior lobe and (5) at the apex and (6) the base of the flocculonodular lobe. All pictures were taken and analyzed at a magnification of 63 times.

PCs were scored as normal or abnormal (Fig. 2). A PC was scored normal when the nucleus was clearly visible, light stained with a clear nucleolus, and the cellular cytoplasm was slightly eosinophilic and uniformly stained. A PC was scored abnormal when the 


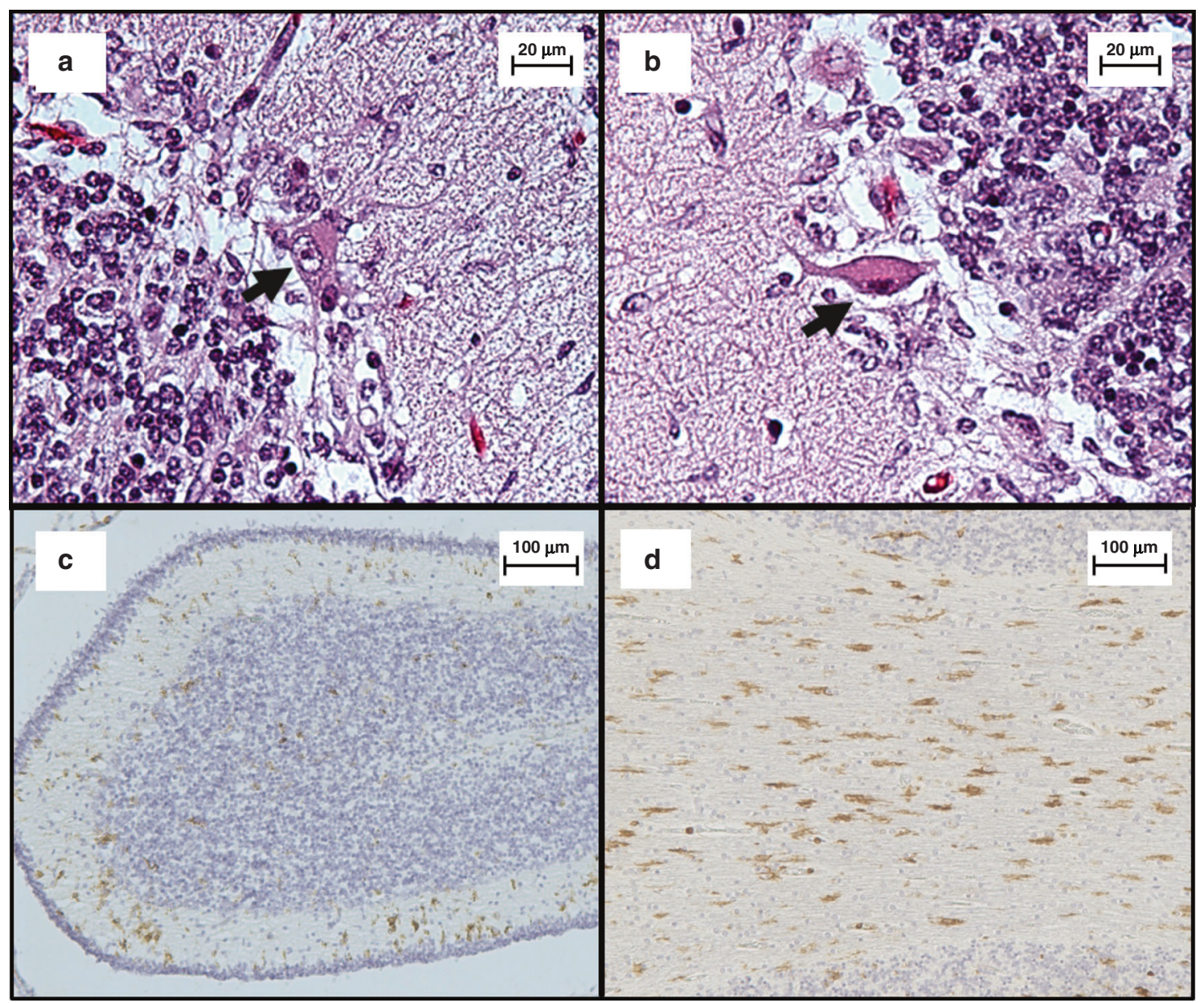

Fig. 2 Examples of histopathology. This figure shows examples of normal PCs (2a) and abnormal PCs (2b) in lobe 2 of the vermis of a patient with severe HIE who died five days after birth and examples of CD68 staining (c) and HLA-DR staining (d).

nucleus was intensively dark stained and shrunken and when there was dark stained, hyper-eosinophilic cytoplasm. PCs were not included in the analyses when we were unable to identify a nucleus, because it was unclear whether this was due to hypoxic-ischemic injury or due to the way the slices were cut.

In every picture the number of normal and abnormal PCs were counted, as well as the total number of PCs. The mean number and percentage for all the analyzed locations within the vermis and hemispheres were used for further analysis. PCs were counted manually and the length of the PC layer was measured using ImageJ (ImageJ program 1.47; National Institutes of Health, USA). The number of PCs per $1000 \mu \mathrm{m}$ is reported in the main text.

\section{H\&E staining-scoring DN}

The DN pictures were taken at three different locations spread throughout the structure, also with a magnification of 63 times. Neurons in the H\&E-stained slices of the DN were also scored normal or abnormal using the same criteria as for PC scoring (see above). Only cells with a somatic diameter of at least $14 \mu \mathrm{m}$ were taken into account, because cell characteristics were not reliably assessable in smaller cells. The DN cell counts (normal, abnormal, and total) were corrected for the measured surface in $0.1 \mathrm{~mm}^{2}$.

H\&E staining-cytotoxic edema

The H\&E-stained slices of the vermis, cerebellar hemispheres, and DN were visually scored for cytotoxic edema. Cytotoxic edema was scored as absent (no, 0 points), minimal, or focal cytotoxic edema (mild, 1 point), diffuse cytotoxic edema (moderate, 2 points), or very extensive cytotoxic edema (severe, 3 points) by an experienced neonatal pathologist.

\section{HLA-DR and CD68 staining}

The slices stained with monoclonal antibodies for HLA-DR and CD68 were scored according to a previously published method. ${ }^{9}$ The slices of the vermis, cerebellar hemispheres, and DN were scored based on the magnification that was necessary to visualize the stained microglia/macrophages: 0 points were given if there was no staining visible (no staining), 1 point when staining was visible with a magnification of $\times 200$ (mild staining), 2 points in case a magnification of $\times 100$ was sufficient to visualize staining (moderate staining), and 3 points if the staining was visible with a magnification of $\times 25$ (severe staining).

\section{Statistical analysis}

Statistical analysis was performed using IBM SPSS Statistics for Windows, version 25 (IBM Armonk, New York, USA). The correlation between the ADC measurements of the two raters was calculated using intraclass correlation coefficient. Mean ADC values between infants with HIE and controls were compared using the independent $t$-test for parametric data and Mann-Whitney $U$ test for non-parametric data. The histopathological measurements (number of normal PC per $1000 \mu \mathrm{m}$ and percentage of normal PC of the total PC) were compared between the different locations in the vermis and hemispheres using 
repeated measures ANOVA (between lobes). The correlation between ADC values and the percentage and count of normal PCs were analyzed with Pearson's $r$ coefficient for parametric and Spearman's rho for non-parametric data. The differences in mean $A D C$ values and count and percentage of normal PCs between the different HLA-DR, CD68, and cytotoxic edema categories were calculated with one-way ANOVA or Kruskal-Wallis $H$ test. $P$ values below 0.05 were considered statistically significant.

\section{RESULTS}

Study population

Thirty-six term neonates with HIE who died and underwent cranial autopsy in the Wilhelmina Children's Hospital between 2000 and 2016 were included in this study. Of those infants, 33 infants were included in the final analysis, two infants were excluded because their antemortem MRI was of poor quality, and one because the postmortem brain tissue was not available from the pathology department. Twenty-two controls were included. Patient characteristics are described and compared in Table 1.
Interrater variability ADC measurements

The interclass correlation of the ADC values between the two different observers was good for the vermis (Cronbach's $a=0.95$, $p<0.001$ ), cerebellar hemispheres (Crohnbach's $a=0.95, p<$ $0.001)$, and DN (Cronbach's $a=0.92, p<0.001)$.

ADC values

Mean ADC values of cerebellar vermis (HIE: $785 \pm 113 \times 10^{-6} \mathrm{~mm}^{2} / \mathrm{s}$; controls: $\left.859 \pm 72 \times 10^{-6} \mathrm{~mm}^{2} / \mathrm{s} ; p=0.009\right)$ and the DN (HIE: $1004 \pm$ $152 \times 10^{-6} \mathrm{~mm}^{2} / \mathrm{s}$; controls: $\left.1140 \pm 82 \times 10^{-6} \mathrm{~mm}^{2} / \mathrm{s} ; \quad p<0.001\right)$ were significantly lower in neonates with HIE compared to controls, but there were no significant differences in ADC values of the cerebellar hemispheres (HIE: $1072 \pm 121 \times 10^{-6} \mathrm{~mm}^{2} / \mathrm{s}$; controls: $1115 \pm 89 \times 10^{-6} \mathrm{~mm}^{2} / \mathrm{s} ; p=0.162$ ) (Fig. 3a, b). ADC values in the infants with HIE were not correlated with postnatal age at MRI.

Histopathology

The count of normal PCs per $1000 \mu \mathrm{m}$ and the percentage of normal PCs were similar in the anterior, posterior, and flocculonodular lobe of the vermis $(p=0.21)$ as well as in the anterior,

\begin{tabular}{|c|c|c|c|c|c|}
\hline Gestational age in weeks, mean (SD) & $39.8(1.6)$ & $40.1(1.3)$ & 0.64 & $39.6(1.5)$ & 0.49 \\
\hline Apgar 1 min, median (IQR) & $1(0-3)$ & $2(1-4)$ & 0.91 & $9(8-9)$ & $p<0.001$ \\
\hline $\mathrm{pH}$, mean (SD) & $6.86(0.19)$ & $7.05(0.19)$ & 0.01 & $\mathrm{n} / \mathrm{a}$ & $\mathrm{n} / \mathrm{a}$ \\
\hline Sarnat, $n(\%)$ & & & 0.52 & & $\mathrm{n} / \mathrm{a}$ \\
\hline Moderate & $7(35.0 \%)$ & $6(46.2 \%)$ & & $\mathrm{n} / \mathrm{a}$ & \\
\hline Severe & $13(65.0 \%)$ & 7 (53.9\%) & & $\mathrm{n} / \mathrm{a}$ & \\
\hline $\begin{array}{l}\text { Predominant type of supratentorial } \\
\text { brain injury, } n(\%)\end{array}$ & & & 0.99 & & $\mathrm{n} / \mathrm{a}$ \\
\hline Field strength MRI, $n(\%)$ & & & $<0.001$ & & 0.002 \\
\hline 1.5 Tesla & $0(0.0 \%)$ & $11(84.6 \%)$ & & $0(0.0 \%)$ & \\
\hline 3.0 Tesla & $20(100.0 \%)$ & $2(15.4 \%)$ & & $22(100.0 \%)$ & \\
\hline $\begin{array}{l}\text { Postnatal age at MRI in days, } \\
\text { median (IQR) }\end{array}$ & $4.0(3.0-4.8)$ & $2.0(1.0-4.5)$ & 0.02 & $7.5(5.0-9.0)$ & $p<0.001$ \\
\hline $\begin{array}{l}\text { Postnatal age at death in days, } \\
\text { median (IQR) }\end{array}$ & $4.5(3.0-5.0)$ & $4.0(2.0-6.0)$ & 0.95 & $\mathrm{n} / \mathrm{a}$ & $\mathrm{n} / \mathrm{a}$ \\
\hline $\begin{array}{l}\text { Days between MRI and death, } \\
\text { median (IQR) }\end{array}$ & $0.0(0.0-1.0)$ & $1.0(0.5-1.0)$ & 0.05 & $\mathrm{n} / \mathrm{a}$ & $\mathrm{n} / \mathrm{a}$ \\
\hline
\end{tabular}
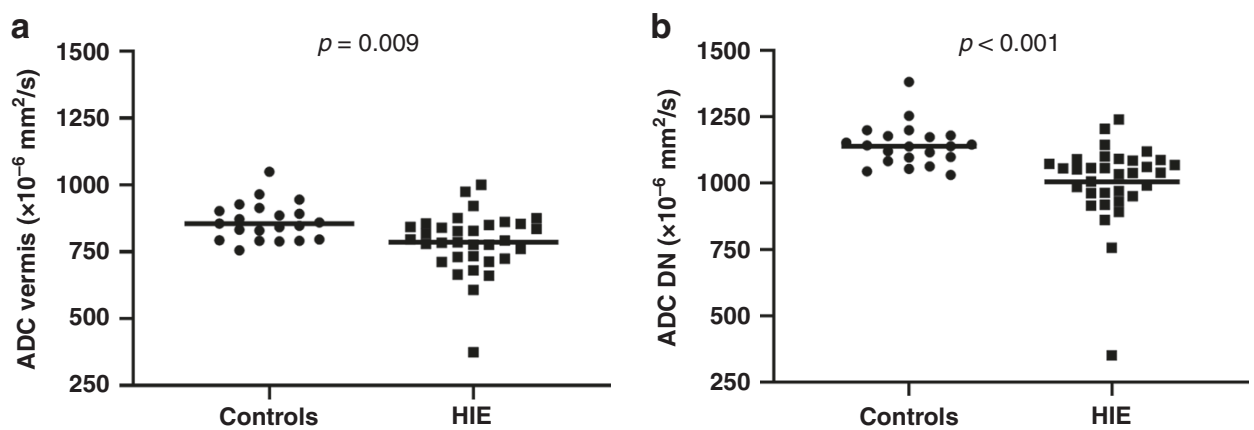

Fig. 3 Differences in ADC values between infants with HIE and controls. Mean ADC values in infants with HIE $(n=33)$ and controls $(n=22)$ in the vermis (a) and the DN (b). 
posterior, and flocculonodular lobe of the cerebellar hemispheres $(p=0.75)$. There was no correlation between the timing of death and number of normal PCs per $1000 \mu \mathrm{m}\left(r_{\mathrm{s}}=0.08, p=0.67\right)$ and percentage of normal PCs $\left(r_{\mathrm{s}}=0.07, p=0.68\right)$.

The median histological cytotoxic edema score was 2 (IQR 1-2) in the vermis, 1 (IQR 0-2) in the cerebellar hemispheres, and 1.5 (IQR 1-2) in the DN. There were no differences in number and percentage of normal PCs between infants with no, mild, moderate, and severe cytotoxic edema in the vermis and cerebellar hemispheres. In the DN, the number of normal neurons $(p=0.001)$ and percentage of normal neurons $(p=0.01)$ were lower in case of more severe cytotoxic edema. Also see Supplementary Fig. 1. The postnatal age of death was not significantly different between infants with no, mild, moderate, and severe cytotoxic edema based on the H\&E-stained slices.

The median CD68 score in the vermis was 2 (IQR 2-3) and HLADR score 2 (IQR 0-3). In the cerebellar hemispheres the median CD68 score was 2 (IQR 2-3) and the median HLA-DR score 1 (IQR $0-3$ ). In the DN the median CD68 score was 2 (IQR 0-3) and HLADR score 0 (IQR 0-3). The count of normal PCs per $1000 \mu \mathrm{m}$ and percentage of normal $\mathrm{PCs}$ in the cerebellar vermis were comparable between the HLA-DR and CD68 categories in the cerebellar vermis. The same applied to the PCs in the hemispheres and the neurons in the DN. Also see Supplementary Fig. 1. Infants with HIE that had more activation of microglia and macrophages did not have more PC injury compared to infants with HIE with less activation.

The postnatal age at death was not significantly different between the different HLA-DR and CD68 categories, except for the CD68 staining in the DN $(p=0.024)$ in which the patients with score 0 died at a median of 5 days after birth and the infants with score 3 at a median of 3 days after birth.

\section{$A D C$ values vs. histopathology}

The ADC values in the vermis were significantly correlated with the amount of normal PC per $1000 \mu \mathrm{m}$ and the percentage of normal PCs in the vermis. In the cerebellar hemispheres and DN there was no correlation between the ADC and the neuron/PC measures (Fig. 4a, c, e).

ADC values in the cerebellar hemispheres, vermis, and DN did not differ between no, mild, moderate, and severe CD68-stained slices of these structures (Fig. 4b, d, f). The same is applied to HLADR staining. The patients with low ADC values in the hemispheres did have severe CD68 staining of the hemisphere and the patient with low ADC values in DN did have severe CD68 and HLA-DR staining of the DN (Fig. 4d, e).

The median ADC values in the vermis were not significantly different between infants with no, mild, moderate, and severe histopathological cytotoxic edema of the vermis $(p=0.08)$. The mean ADC values in the cerebellar hemispheres were also not significantly different between infants no, mild, moderate, and severe histopathological cytotoxic edema of the hemispheres $(p=0.36)$. The ADC values in the DN were comparable between the cytotoxic edema categories in the DN $(p=0.70)$. See Fig. 5.

\section{DISCUSSION}

This study showed that infants with HIE had lower ADC values in the vermis and DN compared to controls, but not in the cerebellar hemispheres. $A D C$ values in the vermis were correlated with the number of normal PCs in the vermis; furthermore, there were no correlations between ADC values and histology measures.

The lower ADC values, suggestive of cytotoxic edema, in the vermis and DN imply that it is possible to detect hypoxic injury in these structures with ADC maps. There were two patients with clear abnormalities on both the DWI and ADC map, so when cytotoxic edema is very severe, the ADC map will detect this. However, in the hemispheres there were no differences between the HIE group and controls; although in case of severe HIE severe cytotoxic edema in the hemispheres is to be expected. ${ }^{9}$ We hypothesize that only parts of the hemispheres show diffusion restriction and that by measuring the whole hemisphere, this effect is levelled out. Though ADC values did not reveal cerebellar injury in the majority of the cases, there was cerebellar histopathological injury in most of the patients. As expected, PC injury was histologically confirmed in the neonates with HIE. PCs fire action potentials at high frequencies of $\sim 100 \mathrm{~Hz}$, and can even achieve frequencies up to $500 \mathrm{~Hz}$ when triggered by climbing fibers. ${ }^{29}$ This high firing rate combined with a large membrane surface sums up to an extraordinary high metabolic demand of PCs and high vulnerability for hypoxic-ischemic injury. ${ }^{30}$ In addition, the high susceptibility of cerebellar PCs for hypoxic-ischemia, as evidenced by the present study, can be explained by their decreased ability to minimize excitotoxic glutamate release in the acute hypoxic-ischemic state. ${ }^{26,31}$

Infants with HIE also showed signs of neuroinflammation, in that we found macrophages and microglia stained with monoclonal antibodies for CD68 and HLA-DR in the cerebellar vermis, hemispheres, and DN. However, PC injury was not associated with the severity of macrophage and microglia activation. This implies that the inflammation is not (solely) caused by PC injury. The PCs are the main output cells of the cerebellum and therefore very important. However, granular cell injury is also described after hypoxic-ischemia and this process might lead to additional inflammation. ${ }^{12,13}$ CD68-stained macrophages and microglia were in an earlier study especially found in the granular layer and white matter and less frequently in the PC layer, which supports this theory. ${ }^{32}$ Another explanation might be that the inflammation markers are time dependent. ${ }^{9,32}$ We did not find an effect of postnatal age at death in our study, except for CD68 staining in the DN, but this effect was the opposite of what we expected based on earlier studies. ${ }^{32}$ The effect of timing between the ischemic insult and death on these CD68 and HLA-DR results cannot be ruled out because of the small sample size. The same is applied for histopathological cytotoxic edema: additional antibodies, such as calbindin, are valuable to further investigate cerebellar injury in the future. ${ }^{12}$

$A D C$ values and histopathological injury of the cerebellum were not correlated, except for ADC values in the vermis and number and percentage of normal PCs. This means that even if the DWI and $A D C$ values are in the reference range, this does not rule out cerebellar injury. An underestimation of cerebellar injury with MRI in neonates with HIE is in agreement with earlier literature. ${ }^{9,25,33,34}$ Kwan et al. ${ }^{25}$ studied 172 infants with HIE that were treated with therapeutic hypothermia and found cerebellar injury in only $4 \%$ of this group using conventional imaging. However, histopathological cerebellar injury was found in $72 \%$ of the 14 infants that underwent autopsy. This study does support that conventional imaging underestimates cerebellar injury even more than DWI. Alderliesten et al. ${ }^{9}$ reported cerebellar abnormalities in $61 \%$ of the patients with HIE based on DWI and in $83 \%$ of the patients based on histopathological analysis. In the same study there was a very good correlation between DWI and histopathology for the supratentorial brain structures. ${ }^{9}$ The reason for the underestimation of cerebellar injury with MRI is not entirely clear. We found that ADC values did not significantly differ between infants with no, mild, moderate, and severe cytotoxic edema based on histopathology. The two patients with severe histopathological cytotoxic edema in the vermis and DN did have very low ADC values. So, cytotoxic edema is only detectable on DWI if the edema is severe and diffuse. Furthermore, histopathological cytotoxic edema was not associated with PC injury, so even without cytotoxic edema there still might be cerebellar injury. Our study suggests that ADC values of the vermis are most reliable; it does show differences between the HIE and control group and it is associated with the count and percentage of normal PCs. When 

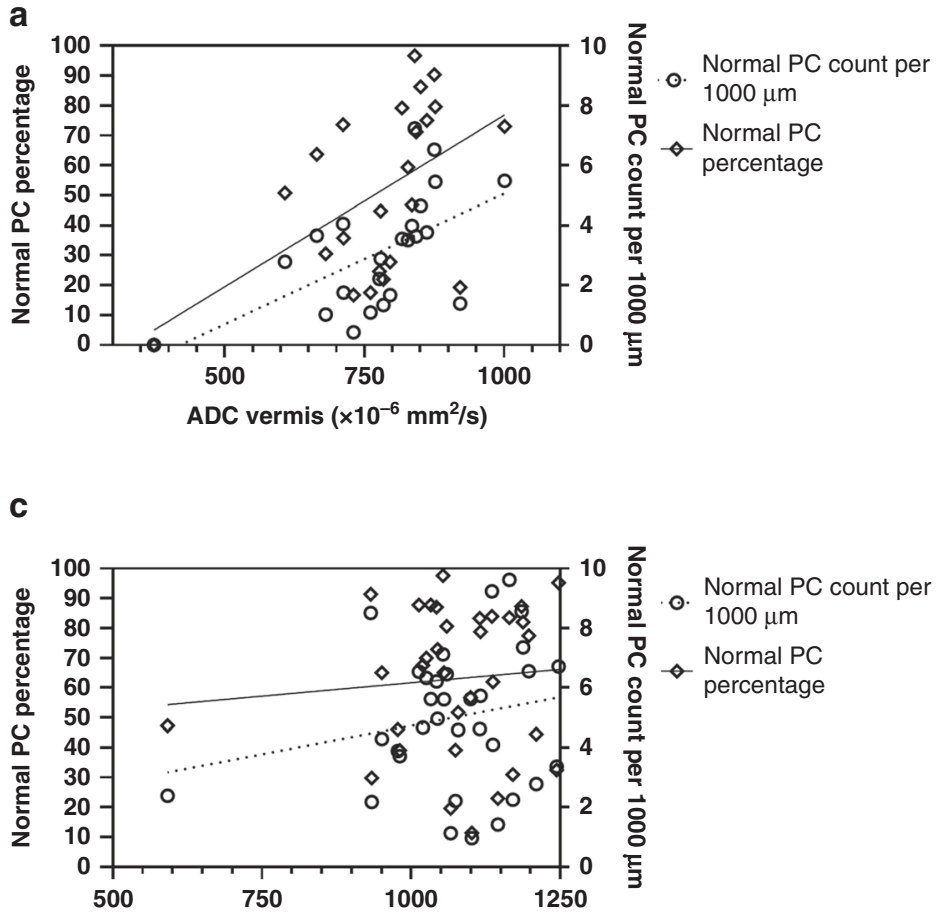

ADC cerebellar hemispheres $\left(\times 10^{-6} \mathrm{~mm}^{2} / \mathrm{s}\right)$

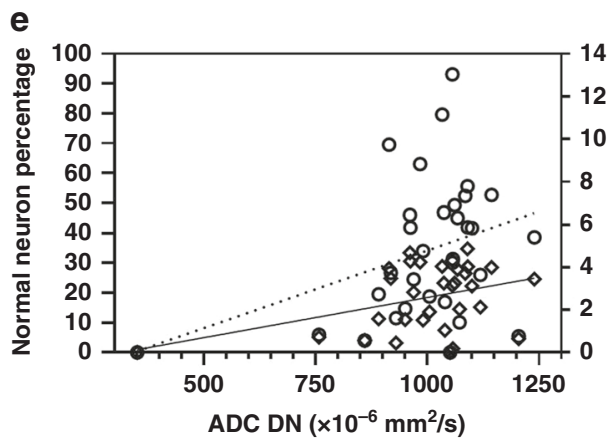

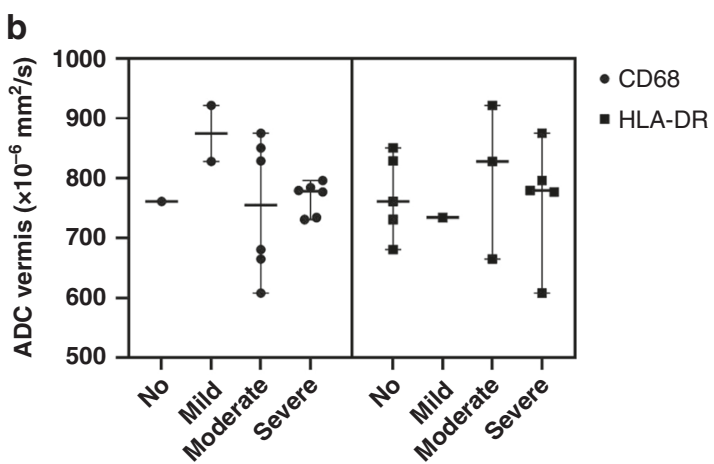
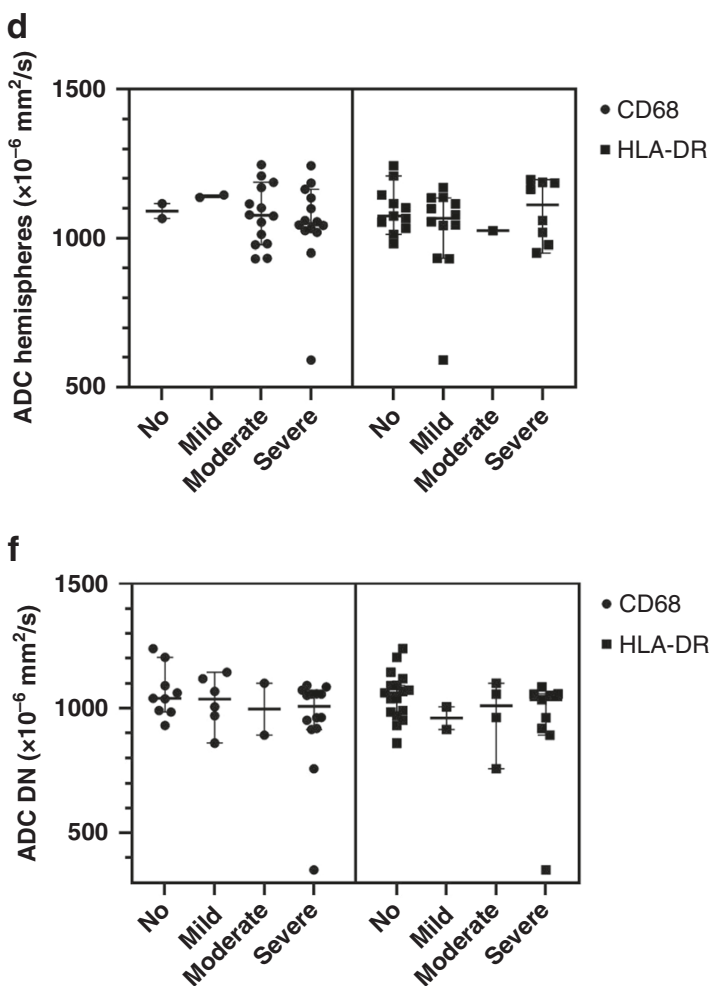

Fig. 4 Comparisons between ADC values and histopathology in infants with HIE. The percentage of normal PCs/neurons is presented at the left $y$-axis and the number of normal PCs/neurons at the right $y$-axis. a ADC values in the vermis were significantly correlated with the count of normal PCs per $1000 \mu \mathrm{m}\left(r_{\mathrm{s}}=0.59, p<0.01\right)$ and the percentage of normal PCs $\left(r_{\mathrm{s}}=0.50, p=0.01\right)$ in the vermis. b ADC values were not different among the CD68 categories $(p=0.39)$ or the HLA-DR categories $(p=0.89)$. c ADC values in the cerebellar hemispheres were not correlated with the count of normal PCs per $1000 \mu \mathrm{m}\left(r_{\mathrm{s}}=0.14, p=0.46\right)$ nor with the percentage of normal PCs $\left(r_{\mathrm{s}}=0.0, p>0.99\right)$ in the hemispheres. d The ADC values in the cerebellar hemispheres were not different among the CD68 categories ( $p=0.59)$ and the HLA-DR categories $(p=0.64)$ in the hemispheres. e ADC values in the DN were not correlated with the count of normal neurons per $0.1 \mathrm{~mm}^{2}$ in the DN $\left(r_{\mathrm{s}}=0.29, p=0.11\right)$ nor with the percentage of normal neurons $\left(r_{\mathrm{s}}=0.25, p=0.17\right)$. $\mathbf{f}$ The ADC values in the DN were comparable between the different CD68 categories $(p=0.46)$ and the HLA-DR categories $(p=0.26)$ in the DN.
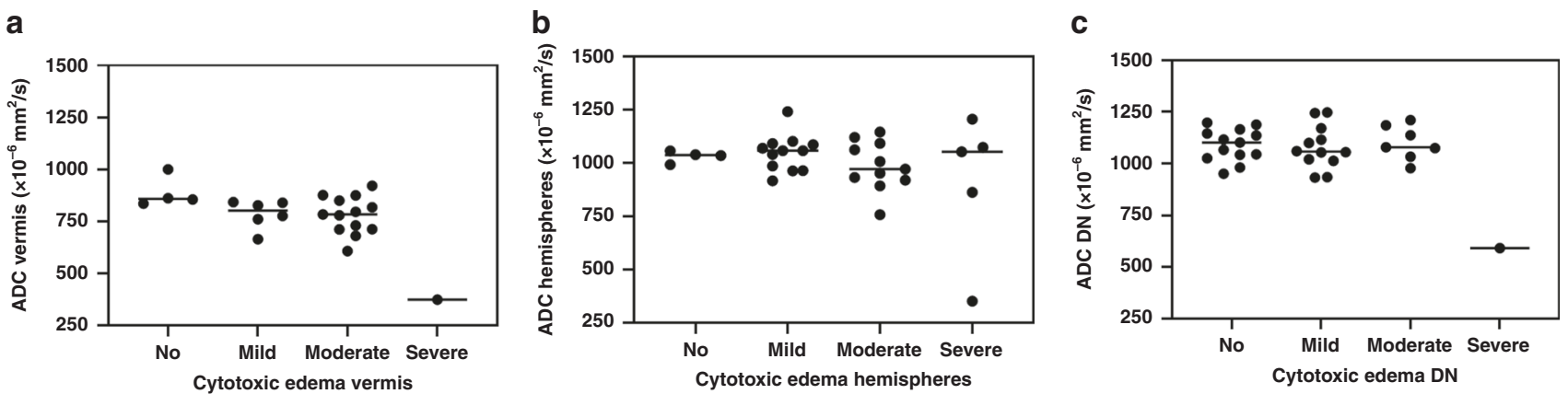

Fig. 5 ADC values versus cytotoxic edema. Median ADC values in the vermis (a), cerebellar hemispheres (b), and DN (c) in infants with no, mild, moderate, and severe cytotoxic edema based on H\&E-stained slices. 
interpreting the $A D C$ values, it is important to realize that pseudonormalization of ADC values occurs about a week after the hypoxic-ischemic event in the cerebrum in infants without therapeutic hypothermia, and a few days later in infants with $\mathrm{TH}^{35}$ The timing of pseudonormalization of ADC values in the cerebellum is unknown. Despite the fact that timing of MRI and death was not significantly associated with the ADC values, the effect of pseudonormalization cannot be ruled out.

Other MR sequences might be more useful to diagnose cerebellar injury in neonates with HIE. For example, Lemmon et al. $^{24}$ showed that fractional anisotropy based on DTI was associated with cerebellar injury and outcome. Including DTI in a future prospective study and comparing this to histopathology would be interesting.

There are some limitations to this study. First, this was a retrospective study, meaning that not all microscopic slices were cut at the same position in the cerebellar hemispheres or vermis and only one slice per structure was available. Also, different protocols for DWI were used. Both might have caused some variability in the results. In addition, the control group of infants with a surgical intervention is not a healthy control group. However, by verifying that their MRI showed no supratentorial or infratentorial abnormalities and their neurocognitive follow-up was normal at 24 months of age, it is justifiable to use this group as a control group. Furthermore, for the histopathological group, it would be useful to have a control group; however, there is no autopsy tissue of healthy term-born neonates available. Finally, our study population was rather small; especially the number of slices of the vermis was limited. A larger study population with sufficient tissue of all patients would have provided more statistical power and would have enabled us to investigate, for instance, the effect of the postnatal age of death on the results. Nevertheless, to the best of our knowledge, this is one of the largest studies to date comparing MRI to histopathology in neonates with HIE. It is a unique dataset with neonatal human postmortem tissue and we were able to use quantitative MRI measurements, which was proven to be reproducible by different raters.

Future research should focus on elaborate staining of the cerebellum to study the injury in infants with HIE in more depth. Additionally, it would be interesting to explore the pattern of PC injury in the cerebellum in more detail. Finally, more research is needed to study the association between ADC values of the cerebellum and neurocognitive outcome. Prospective research is necessary to answer this question.

\section{CONCLUSIONS}

In conclusion, ADC values were lower in the vermis and DN of infants with HIE compared to controls, but similar in the cerebellar hemispheres. Only very severe cytotoxic edema can be detected with $A D C$ measurements. $A D C$ values in the vermis were significantly correlated with PC injury; therefore, abnormal ADC values in the vermis are in the current study, the most representative for histopathological injury. Furthermore, there were no correlations between ADC values and histopathological measures, though histopathological injury was found in all quantified cerebellar structures, implying that normal ADC values do not guarantee a lack of injury to the cerebellum.

\section{ACKNOWLEDGEMENTS}

The authors thank Liska Scheffers and Benthe Bogers for their help with the histopathological analyses. The Ph.D. position of K.V.A. is funded by the Horizon 2020 research and innovation Program of the European Union (call H2020-PHC-2015-twostage, grant 667224, ALBINO).

\section{AUTHOR CONTRIBUTIONS}

K.V.A. has substantially contributed to conception and design of the study, acquisition, analysis and interpretation of the data, and drafting the paper. L.M. has substantially contributed to acquisition, analysis, and interpretation of the data and drafting the paper. N.v.d.A., C.N., L.d.V., and M.B. have substantially contributed to interpretation of the data and revised the article critically for important intellectual content. T.A. has substantially contributed to acquisition and interpretation of the data and revised the article critically for important intellectual content. P.N. and J.D. have substantially contributed to conception and design of the study, acquisition and interpretation of the data, and revised the article critically for important intellectual content. F.G. has substantially contributed to analysis and interpretation of the data and revised the article critically for important intellectual content. F.H. has substantially contributed to conception and design of the study, interpretation of the data, and revised the article critically for important intellectual content.

\section{ADDITIONAL INFORMATION}

The online version of this article (https://doi.org/10.1038/s41390-020-01173-z) contains supplementary material, which is available to authorized users.

Competing interests: F.G. is expert witness in cases of perinatal asphyxia. The other authors have no conflict of interest to declare.

Publisher's note Springer Nature remains neutral with regard to jurisdictional claims in published maps and institutional affiliations.

\section{REFERENCES}

1. Azzopardi, D. et al. Effects of hypothermia for perinatal asphyxia on childhood outcomes. N. Engl. J. Med. 371, 140-149 (2014).

2. van Handel, M., de Sonneville, L., de Vries, L. S., Jongmans, M. J. \& Swaab, H. Specific memory impairment following neonatal encephalopathy in term-born children. Dev. Neuropsychol. 37, 30-50 (2012).

3. van Handel, M., Swaab, H., de Vries, L. S. \& Jongmans, M. J. Behavioral outcome in children with a history of neonatal encephalopathy following perinatal asphyxia. J. Pediatr. Psychol. 35, 286-295 (2010).

4. De Vries, L. S. \& Jongmans, M. J. Long-term outcome after neonatal hypoxicischaemic encephalopathy. Arch. Dis. Child Fetal Neonatal Ed. 95, 220-225 (2010).

5. Miller, S. P. et al. Patterns of brain injury in term neonatal encephalopathy. J. Pediatr. 146, 453-460 (2005).

6. Groenendaal, F. \& de Vries, L. S. Fifty years of brain imaging in neonatal encephalopathy following perinatal asphyxia. Pediatr. Res. 81, 150-155 (2017).

7. Goergen, S. K. et al. Early MRI in term infants with perinatal hypoxic-ischaemic brain injury: interobserver agreement and MRI predictors of outcome at 2 years. Clin. Radiol. 69, 72-81 (2014).

8. Robertson, R. L. \& Glasier, C. M. Diffusion-weighted imaging of the brain in infants and children. Pediatr. Radiol. 37, 749-768 (2007).

9. Alderliesten, T., Nikkels, P. G. J., Benders, M. J. N. L., De Vries, L. S. \& Groenendaal, F. Antemortem cranial MRI compared with postmortem histopathologic examination of the brain in term infants with neonatal encephalopathy following perinatal asphyxia. Arch. Dis. Child Fetal Neonatal Ed. 98, F304-9 (2013).

10. Alderliesten, T., De Vries, L. S., Benders, M. J. N. L., Koopman, C. \& Groenendaal, F. MR imaging and outcome of term neonates with perinatal asphyxia: value of diffusion-weighted MR imaging and ${ }^{1} \mathrm{H}$ MR spectroscopy. Radiology 261, 235-242 (2011).

11. Alderliesten, T. et al. MRI and spectroscopy in (near) term neonates with perinatal asphyxia and therapeutic hypothermia. Arch. Dis. Child Fetal Neonatal Ed. 102, F147-F152 (2017).

12. Campanille, V. et al. Long lasting cerebellar alterations after perinatal asphyxia in rats. Brain Res. Bull. 116, 57-66 (2015).

13. Biran, V., Verney, C. \& Ferriero, D. M. Perinatal cerebellar injury in human and animal models. Neurol. Res. Int. 2012, 1-9 (2012).

14. Wang, V. Y. \& Zoghbi, H. Y. Genetic regulation of cerebellar development. Nat. Rev. Neurosci. 2, 484-491 (2001).

15. Rice, D. \& Barone, S. Critical periods of vulnerability for the developing nervous system: evidence from humans and animal models. Environ. Health Perspect. 108, 511-533 (2000).

16. Zervas, M., Blaess, S. \& Joyner, A. L. Classical embryological studies and modern genetic analysis of midbrain and cerebellum development. Curr. Top. Dev. Biol. 69, 101-138 (2005).

17. Sotelo, C. Cellular and genetic regulation of the development of the cerebellar system. Prog. Neurobiol. 72, 295-339 (2004).

18. Sarna, J. R. \& Hawkes, R. Patterned Purkinje cell death in the cerebellum. Prog. Neurobiol. 70, 473-507 (2003). 
19. Kemp, K. C. et al. Purkinje cell injury, structural plasticity and fusion in patients with Friedreich's ataxia. Acta Neuropathol. Commun. 4, 53 (2016).

20. Allen, G. \& Courchesne, E. Differential effects of developmental cerebellar abnormality on cognitive and motor functions in the cerebellum: an fMRI study of autism. Am. J. Psychiatry 160, 262-273 (2003).

21. Le Strange, E., Saeed, N., Cowan, F. M., Edwards, A. D. \& Rutherford, M. A. MR imaging quantification of cerebellar growth following hypoxic-ischemic injury to the neonatal brain. Am. J. Neuroradiol. 25, 463-468 (2004).

22. Wang, S. S. H., Kloth, A. D. \& Badura, A. The cerebellum, sensitive periods, and autism. Neuron 83, 518-532 (2014).

23. Hortensius, L. M. et al. Neurodevelopmental consequences of preterm isolated cerebellar hemorrhage: a systematic review. Pediatrics 142, e20180609 (2018).

24. Lemmon, M. E. et al. Diffusion tensor imaging detects occult cerebellar injury in severe neonatal hypoxic-ischemic encephalopathy. Dev. Neurosci. 39, 207-214 (2017).

25. Kwan, S. et al. Injury to the cerebellum in term asphyxiated newborns treated with hypothermia. Am. J. Neuroradiol. 36, 1542-1549 (2015)

26. Hausmann, R., Seidl, S. \& Betz, P. Hypoxic changes in Purkinje cells of the human cerebellum. Int J. Leg. Med. 121, 175-183 (2007).

27. Zhang, Y., Magnus, G. \& Han, V. Z. Cell type-specific plasticity at parallel fiber synapses onto Purkinje cells in the posterior caudal lobe of the mormyrid fish cerebellum. J. Neurophysiol. 120, 644-661 (2018).
28. Dum, R. P. \& Strick, P. L. An unfolded map of the cerebellar dentate nucleus and its projections to the cerebral cortex. J. Neurophysiol. 89, 634-639 (2003).

29. Khaliq, Z. M. \& Raman, I. M. Axonal propagation of simple and complex spikes in cerebellar Purkinje neurons. J. Neurosci. 25, 454-463 (2005).

30. Witter, L. et al. Purkinje cell collaterals enable output signals from the cerebellar cortex to feed back to Purkinje cells and interneurons. Neuron 91, 312-319 (2016).

31. Welsh, J. P. et al. Why do Purkinje cells die so easily after global brain ischemia? Aldolase C, EAAT4, and the cerebellar contribution to posthypoxic myoclonus. Adv. Neurol. 89, 331-359 (2002).

32. Groenendaal, F., Lammers, H., Smit, D. \& Nikkels, P. G. J. Nitrotyrosine in brain tissue of neonates after perinatal asphyxia. Arch. Dis. Child Fetal Neonatal Ed. 91, 429-433 (2006).

33. Vermeulen, R. J. et al. Diffusion-weighted MRI in severe neonatal hypoxic ischaemia: the white cerebrum. Neuropediatrics 34, 72-76 (2003).

34. Liauw, L., Van Wezel-Meijler, G., Veen, S., Van Buchem, M. A. \& Van Der Grond, J. Do apparent diffusion coefficient measurements predict outcome in children with neonatal hypoxic-ischemic encephalopathy? Am. J. Neuroradiol. 30, 264-270 (2009).

35. Bednarek, N. et al. Impact of therapeutic hypothermia on MRI diffusion changes in neonatal encephalopathy. Neurology 78, 1420-1427 (2012). 